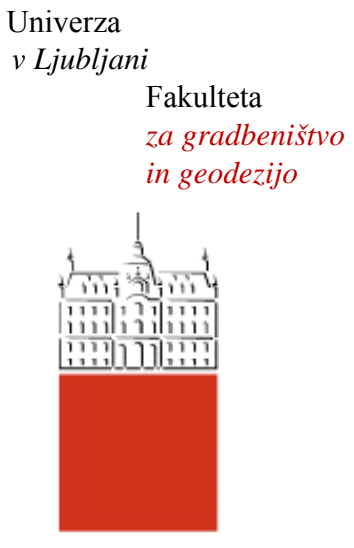

Jamova cesta 2

1000 Ljubljana, Slovenija

http://www3.fgg.uni-lj.si/

\section{DRUGG - Digitalni repozitorij UL FGG http://drugg.fgg.uni-lj.si/}

Ta članek je avtorjeva zadnja recenzirana različica, kot je bila sprejeta po opravljeni recenziji.

Prosimo, da se pri navajanju sklicujte na bibliografske podatke, kot je navedeno:
University
of Ljubljana

Faculty of

Civil and Geodetic

Engineering

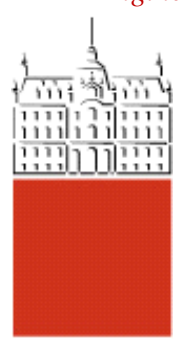

Jamova cesta 2

SI - 1000 Ljubljana, Slovenia

http://www3.fgg.uni-lj.si/en/

DRUGG - The Digital Repository http://drugg.fgg.uni-lj.si/

This version of the article is author's manuscript as accepted for publishing after the review process.

When citing, please refer to the publisher's bibliographic information as follows:

Schnabl, S., Planinc, I. 2011. Inelastic buckling of two-layer composite columns with nonlinear interface compliance. International Journal of Mechanical Sciences 53-12: 1077-1083 DOI: $10.1016 /$ j.jimecsci.2011.09.002. 


\title{
Inelastic buckling of two-layer composite columns with non-linear interface compliance
}

\author{
S. Schnabl* and I. Planinc \\ University of Ljubljana, Faculty of Civil and Geodetic Engineering, Jamova 2, \\ SI-1115 Ljubljana, Slovenia
}

\begin{abstract}
A mathematical model for a slip-buckling problem has been proposed and its exact solution has been found for the analysis of materially inelastic two-layer composite columns with non-linear interface compliance. The mathematical model has been carried out to evaluate exact critical buckling loads. It has been demonstrated mathematically exactly, that exact critical buckling loads are influenced by the initial stiffness, and hence on linear portion of the interface force-slip relationship. Besides, it has been shown that material inelasticity can reduce the critical buckling loads significantly and that the interlayer stiffness has an important effect on the transition between the elastic and inelastic buckling.
\end{abstract}

Keywords: stability, buckling, inelastic, composite, slip, exact.

\footnotetext{
*Corresponding author. Tel.: +38614768 615; Fax: +386 14768629

E-mail address: sschnabl@fgg.uni-lj.si (S. Schnabl)
} 


\section{Introduction}

Layered columns consisting of different or like materials are frequently encountered in a wide range of applications. Due to their high strength-to weight and stiffness-to-weight ratios, slender composite columns are widely used in in aerospace engineering, structural engineering, shipbuilding, and in other branches of industry. In the field of structural engineering typical examples of aforementioned layered structures are steel-concrete and timber-concrete composite columns, layered timber columns, sandwich columns, concrete columns externally reinforced with laminates, and many more. The behaviour of these structures largely depends on the type of the connection between the layers and the quality of the used materials.

Since absolutely stiff connection between the layers cannot be achieved in practice, an inter-layer slip between these layers develops, which significantly can affect the mechanical behaviour of layered structure. Accordingly, the inter-layer slip has to be taken into consideration in what is called partial interaction analysis of composite structures. Consequently, many published papers that take into account the inter-layer slip analytically or numerically are available in the literature. No attempt is made to discuss it here but the interested reader is referred to the, e.g. [1-14].

The strength of straight layered columns depends to a great extent on their stability and cohesion between the layers. It is therefore of practical importance to derive exact solutions for such problems. To date only a few exact slip-buckling models of composite columns have been developed; see, for example, [15-20]. 
In all these previous exact investigations, linear stress-strain relations of the material and linear interfacial constitutive laws between the layers have been assumed. Actually, in reality a highly non-linear material and force-slip law of the interface can be obtained. Notwithstanding, to the best of the authors' knowledge, there exist relatively few exact solutions where linear material an idealized bilinear force-slip law of the interface has been assumed, see, for example [21], but no exact investigation of slip-buckling problem where general inealstic material and non-linear interfacial constitutive law would be employed.

Therefore, the main objective of this paper is to develop a mathematical model for a slip-buckling analysis of geometrically perfect materially inelastic twolayer composite columns with non-linear interfacial compliance between the layers. In many cases, the compliant layer between the layers has been considered to be a very thin interphase layer with vanishing thickness; referred to in the literature as an interface. The interface arises from the damage in composites, e.g. debonding, sliding and cracking across the interface. In this paper, a non-linear tangentially compliant interface layer with non-zero thickness $d$ is assumed to occupy the area between the layers. The thickness of the interface layer depends on the mechanical properties of the composite, and must be determined experimentally. The mechanical behaviour of the interface is described by the general nonuniform, non-linear interface constitutive law.

Exact critical buckling loads of geometrically perfect materially inelastic twolayer composite columns with non-linear compliant interfaces are derived using the linearized stability theory [22]. Therefore, the exact critical buckling forces are determined from the solution of a linear eigenvalue problem, i.e., $\operatorname{det} \mathbf{K}_{\mathbf{t}}=$ 0 (see, [25]). 
In the numerical examples, the proposed exact formulation of the inelastic slipbuckling problem is used to investigate the influence of a non-linear interface compliance and material inelasticity on buckling forces of geometrically perfect two-layer composite columns with interlayer slip between the layers.

\section{Formulation of an inelastic slip-buckling problem}

\subsection{Assumptions}

An exact inelastic slip-buckling formulation of the planar two-layer composite column with compliant interface between the layers used in this paper is based upon the following basic assumptions: (1) the individual layer of the composite column is geometrically perfect and straight; (2) the axial load is loaded eccentrically at a distance $e$ from the reference axis of the two-layer composite column in such a way that a homogeneous stress-strain state at primary configurations is obtained; (3) the material of layers is inelastic; (4) displacements, strains and rotations are finite (each of the layers satisfies the assumptions of geometrically exact Reissner beam theory); (5) the effect of shear deformation is not taken into account; (6) the "Bernoulli hypothesis" of linear distribution of strains over each layer is assumed; (7) the layers are continuously connected through the compliant interface which obey a general nonuniform, non-linear constitutive force-slip law; (8) shapes of the cross-sections are symmetric with respect to the plane of deformation and remain unchanged in the form and size during deformation; (9) an interlayer slip can occur at the interface between the layers, but no transverse separation (uplift) between them is possible.

\subsection{Governing equations}

We consider an initially straight, planar, two-layer composite column of undeformed length $L$ with. Layers as shown in Fig. 1 are marked by letters $a$ 
and $b$. A compliant interface layer with its thickness $d$ takes a position between the layers $a$ and $b$. The column is placed in the $(X, Z)$ plane of spatial Cartesian coordinate system with coordinates $(X, Y, Z)$ and unit base vectors $\mathbf{E}_{X}, \mathbf{E}_{Y}$, and $\mathbf{E}_{Z}=\mathbf{E}_{X} \times \boldsymbol{E}_{Y}$. The undeformed reference axis of the layered column is parametrized by the undeformed arc-length $x$. Local coordinate system $(x, y, z)$ is assumed to coincide initially with spatial coordinates, and then it follows the deformation of the column. The geometrically perfect composite column is subjected to a conservative compressive axial force $P$ centrally located at both ends in such way that homogeneous strain and stress state at primary configuration of the column is achieved. For further details an interested reader is referred to e.g., [13, 16, 19].

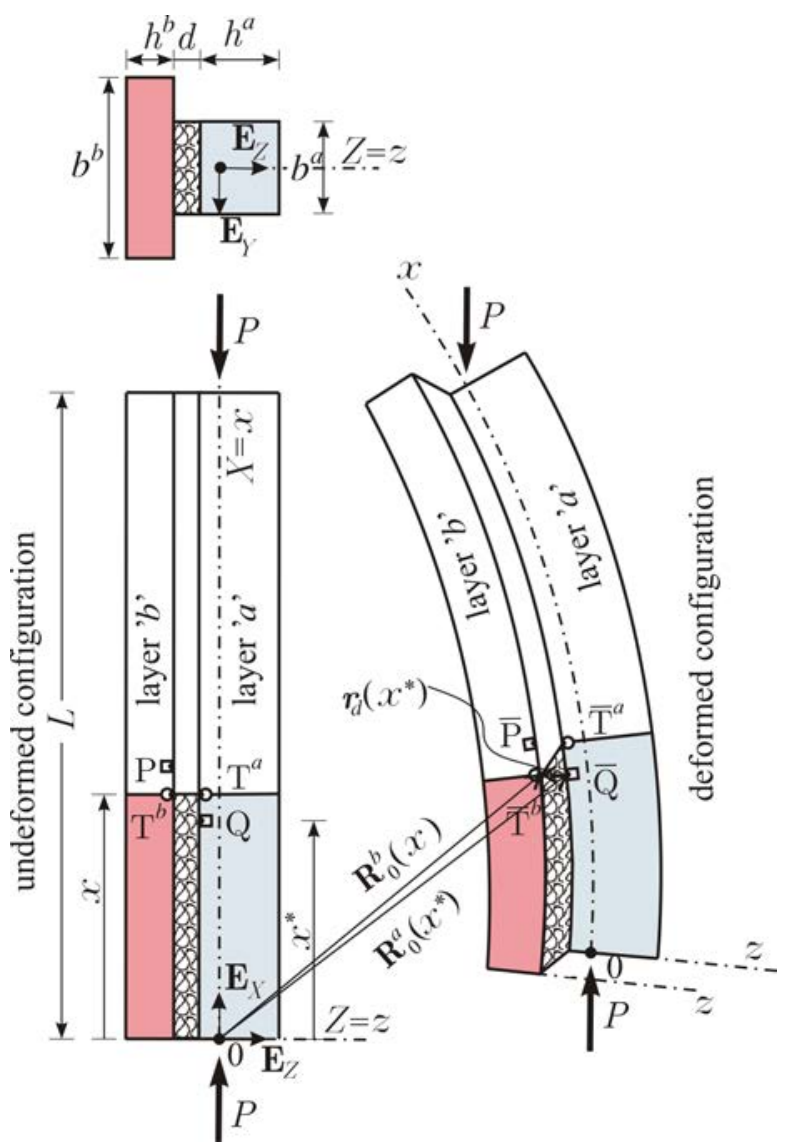

Figure 1. Geometry and notation for a straight geometrically perfect two-layer composite column with an interface layer between the layers. 


\subsubsection{Kinematic equations}

The deformed configurations of the reference axes of layers $a$ and $b$ are defined by vector-valued functions (see Fig. 1)

$$
\mathbf{R}_{0}^{i}=X^{i} \mathbf{E}_{X}+Y^{i} \mathbf{E}_{Y}+Z^{i} \mathbf{E}_{Z}=\left(x^{i}+u^{i}\right) \mathbf{E}_{X}+y^{i} \mathbf{E}_{Y}+w^{i} \mathbf{E}_{Z},
$$

where superscript $i=a, b$ denotes that quantities are related to layer $a$ and $b$, respectively. Functions $u^{i}$ and $w^{i}$ denote the components of the displacement vector of layer $i$ at the reference axis with respect to the base vectors $\mathbf{E}_{X}$ and $\mathbf{E}_{Z}$. The geometrical components $u^{i}$ and $w^{i}$ of the the vector-valued functions $\mathbf{R}_{0}^{i}$ are related to the deformation variables with the equations derived by [26]:

$$
\begin{gathered}
1+u^{i \prime}-\left(1+\varepsilon^{i}\right) \cos \varphi^{i}=0, \\
w^{i \prime}+\left(1+\varepsilon^{i}\right) \sin \varphi^{i}=0, \\
\varphi^{i \prime}-\kappa^{i}=0 .
\end{gathered}
$$

Here, the prime $(\bullet)^{\prime}$ denotes the derivative with respect to $x$. In $(2)$, the deformation variables $\varepsilon^{i}$ are the extensional strains of the reference axes of layers $a$ and $b ; \kappa^{i}$ are the pseudocurvatures [27]; whereas $\varphi^{i}$ are the rotations of layers' reference axes.

\subsubsection{Equilibrium equations}

The composite column is subjected to a force $P$ at both ends. Furthermore, each layer of the two-layer composite column is subjected to interlayer contact tractions measured per unit of layer's undeformed length. In order to write constitutive equations in usually used coordinate system, it is suitable to express the $(X, Z)$ components of the interlayer contact tractions with the tangential and normal components of the interlayer tractions $p_{t}^{i}$ and $p_{n}^{i}$ (see Fig. 2): 


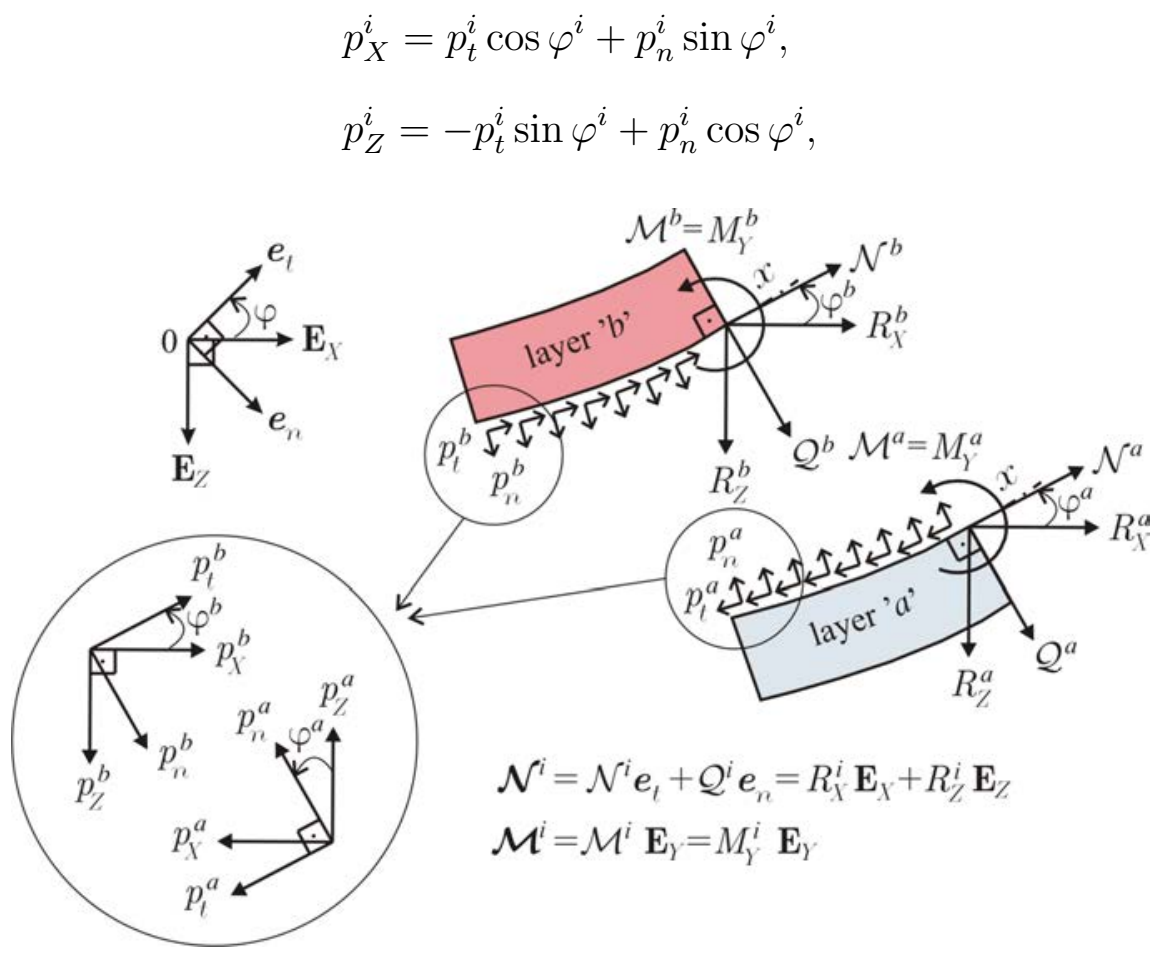

Figure 2. Interlayer contact tractions and generalized equilibrium internal forces and moments with respect to the fixed global and rotated local coordinate system.

Using (3), the equilibrium equations of each layer are, see e.g. [16, 19, 26]:

$$
\begin{gathered}
R_{X}^{i \prime}+p_{X}^{i}=R_{X}^{i \prime}+p_{t}^{i} \cos \varphi^{i}+p_{n}^{i} \sin \varphi^{i}=0, \\
R_{Z}^{i \prime}+p_{Z}^{i}=R_{Z}^{i \prime}-p_{t}^{i} \sin \varphi^{i}+p_{n}^{i} \cos \varphi^{i}=0, \\
M_{Y}^{i \prime}-\left(1+\varepsilon^{i}\right) \mathcal{Q}^{i}+m_{Y}^{i}=0,
\end{gathered}
$$

where

$$
\begin{gathered}
\mathcal{N}^{i}=R_{X}^{i} \cos \varphi^{i}-R_{Z}^{i} \sin \varphi^{i}, \\
\mathcal{Q}^{i}=R_{X}^{i} \sin \varphi^{i}+R_{Z}^{i} \cos \varphi^{i}, \\
\mathcal{M}^{i}=M_{Y}^{i} .
\end{gathered}
$$

$R_{X}^{i}, R_{Z}^{i}$, and $M_{Y}^{i}$ in (4)-(5) represent the generalized equilibrium internal forces and moments of a cross-section of layers $a$ and $b$ with respect to the fixed coordinate basis. On the other hand, $\mathcal{N}^{i}$ and $\mathcal{Q}^{i}$ represent the equilibrium 
axial and shear internal forces of the layers' cross-sections with respect to the rotated local coordinate system. Functions $\mathcal{M}^{i}$ are the equilibrium bending moments.

\subsubsection{Constitutive equations}

To relate the equilibrium internal forces $\mathcal{N}^{i}$ and $\mathcal{Q}^{i}$, and equilibrium internal moments $\mathcal{M}^{i}$ to a material model, the following set of equations which assure the balance of equilibrium and constitutive cross-sectional forces and bending moments of the composite column have been introduced. Due to the assumption that the transverse shear deformations are neglected, the constitutive equations of a two-layer composite column are

$$
\begin{aligned}
\mathcal{N}^{i}-\mathcal{N}_{C}^{i}\left(D^{i}\right) & =\mathcal{N}^{i}-\int_{\mathcal{A}^{i}} \sigma_{C}^{i}\left(D^{i}\right) \mathrm{d} A^{i}=0 \\
\mathcal{M}^{i}-\mathcal{M}_{C}^{i}\left(D^{i}\right) & =\mathcal{M}^{i}-\int_{\mathcal{A}^{i}} z \sigma_{C}^{i}\left(D^{i}\right) \mathrm{d} A^{i}=0 .
\end{aligned}
$$

The constitutive functions $\mathcal{N}_{C}^{i}$ and $\mathcal{M}_{C}^{i}$ introduced in (6) are dependent only on deformation variables $\varepsilon^{i}$ and $\kappa^{i}$ and are subordinated to the adopted inelastic constitutive model defined by the uniaxial true stress-strain relations

$$
\sigma_{C}^{i}\left(D^{i}\right)=\mathcal{F}^{i}\left(\varepsilon^{i}+z \kappa^{i}\right)
$$

where $\sigma_{C}^{i}$ are the true extensional stresses of layers $a$ and $b ; D^{i}$ are the mechanical extensional strains in longitudinal direction in layers $a$ and $b$; and $\mathcal{F}^{i}$ are experimentally determined non-linear functions that describe a broad class of materials. Moreover, the contact constitutive law must also be introduced. In general, a non-linear constitutive law of bond slip between the layers is present. Therefore, in the present analysis, a general nonuniform, non-linear constitutive force-slip law of the interface between the layers is employed:

$$
p_{t}^{a}=\mathcal{H}(\Delta)
$$


where $\mathcal{H}$ is a non-linear function dependent on the type of the connection.

\subsubsection{Constraining equations}

Once the layers are connected, the upper layer $b$ is constrained to follow the deformation of the lower layer $a$ and vice versa. As already stated, the layers can slip along each other but their transverse separation (uplift) or penetration is not allowed. This fact is expressed by a kinematic-constraint requirement

$$
\mathbf{R}_{0}^{b}(x)=\mathbf{R}_{0}^{a}\left(x^{*}\right)+\mathbf{r}_{d}\left(x^{*}\right),
$$

where $x$ and $x^{*}$ are coordinates of two distinct particles $\bar{T}^{b}$ and $\bar{Q}$ of layers $b$ and $a$ in the undeformed configuration which are, in the deformed configuration, related to each other by a vector-valued function $\boldsymbol{r}_{d}\left(x^{*}\right)=-d \boldsymbol{e}_{n}\left(x^{*}\right)$, (see, Fig. 1). Eq. (9) can be written equivalently in componential form as

$$
\begin{aligned}
x+u^{b}(x) & =x^{*}+u^{a}\left(x^{*}\right)-d \sin \varphi^{a}\left(x^{*}\right), \\
w^{b}(x) & =w^{a}\left(x^{*}\right)-d \cos \varphi^{a}\left(x^{*}\right) .
\end{aligned}
$$

By differentiating first equation of (10), adding the results with (2), the following direct relation between the differentials of material coordinates $x$ and $x^{*}$ is obtained

$$
\frac{\mathrm{d} x^{*}}{\mathrm{~d} x}=\frac{\left(1+\varepsilon^{b}(x)\right) \cos \varphi^{b}(x)}{\left(1+\varepsilon^{a}\left(x^{*}\right)-d \kappa^{b}\left(x^{*}\right)\right) \cos \varphi^{b}\left(x^{*}\right)} .
$$

Using (11), and taking into account the fact that the rotations of layers are identical (see, [16])

$$
\varphi^{a}\left(x^{*}\right)=\varphi^{b}(x)
$$

it can be shown that the pseudocurvatures of layers $a$ and $b$ are constrained to each other by

$$
\kappa^{a}\left(x^{*}\right) \frac{1+\varepsilon^{b}(x)}{1+\varepsilon^{a}\left(x^{*}\right)-d \kappa^{a}\left(x^{*}\right)}=\kappa^{b}(x)
$$


The slip that occurs between the two particles of layers $a$ and $b$ which are in the contact through the interphase layer in the undeformed configuration is denoted by $\Delta$ and defined as

$$
\Delta^{\prime}=\varepsilon^{a}-\varepsilon^{b}-d \kappa^{b}
$$

In addition to the above presented constraining equations, from the third Newton's law, an equilibrium of the interlayer contact tractions of the particles in contact is expressed in the vector-valued function form as

$$
\mathbf{p}^{a}+\mathbf{p}^{b}=\mathbf{0}
$$

and, by substituting (3) to (15), in componential form as

$$
\begin{aligned}
p_{t}^{a} \cos \varphi^{a}+p_{n}^{a} \sin \varphi^{a}+p_{t}^{b} \cos \varphi^{b}+p_{n}^{b} \sin \varphi^{b} & =0, \\
-p_{t}^{a} \sin \varphi^{a}+p_{n}^{a} \cos \varphi^{a}-p_{t}^{b} \sin \varphi^{b}+p_{n}^{b} \cos \varphi^{b} & =0 .
\end{aligned}
$$

Consequently, a complete set of non-linear governing equations of a two-layer composite column, Eqs. (2), (4)-(6), (8), (10), (14), and (16) consists of 28 equations for 28 unknown functions: $u^{i}, w^{i}, \varphi^{i}, \varepsilon^{i}, \kappa^{i}, R_{X}^{i}, R_{Z}^{i}, M_{Y}^{i}, \mathcal{N}^{i}, \mathcal{Q}^{i}$, $\mathcal{M}^{i}, p_{t}^{i}, p_{n}^{i}, \Delta$, and, $x^{*}$.

\subsection{Linearized equations}

In order to investigate the stability of non-linear boundary value problems the approximation methods should be used. In this paper, a linearized theory of stability is used. This theory is based on the fact that the bifurcation points of the non-linear system coincide with the critical points of its equivalent linearized system [22]. The application of the linearized stability theory, regarding the existence and uniqueness of the solution of Reissner's elastica, is presented by Flajs et al. [23]. 
The above-mentioned linearized theory of stability is based upon the variation of a functional $\mathcal{F}$, which will here be made in the sense of the continuous linear Gateaux operator or directional derivative, defined as follows [24]

$$
\delta \mathcal{F}(\mathbf{x}, \delta \mathbf{x})=\lim _{\alpha \rightarrow 0} \frac{\mathcal{F}(\mathbf{x}+\alpha \delta \mathbf{x})-\mathcal{F}(\mathbf{x})}{\alpha}=\left.\frac{\mathrm{d}}{\mathrm{d} \alpha}\right|_{\alpha=0} \mathcal{F}(\mathbf{x}+\alpha \delta \mathbf{x}),
$$

where the $\mathbf{x}$ and $\delta \mathbf{x}$ represent the generalized displacement field and its increment, respectively, and $\alpha$ is an arbitrary small scalar parameter. $\delta \mathcal{F}(\mathbf{x}, \delta \mathbf{x})$ is also called linearization or linear approximation of $\delta \mathcal{F}$ at $\mathbf{x}$. Accordingly, it is convenient for Eqs. (2), (4)-(6), (8), (10), (14), and (16) to be re-written in compact form as $\mathcal{F}=\left\{\mathcal{F}_{1}, \mathcal{F}_{1}, \ldots, \mathcal{F}_{28}\right\}^{T}$, and their arguments as $\mathbf{x}=$ $\left\{u^{i}, w^{i}, \ldots, p_{n}^{i}, \Delta, x^{*}\right\}^{T}$.

After the linearization of the governing Eqs. (2), (4)-(6), (8), (10), (14), and (16) has been completed, the linearized equations of the two-layer composite column buckling problem have to be evaluated at the primary configuration of the column. The primary configuration of the column is here defined as the configuration in which the composite column, which is subjected to the point force $P$, remains straight:

$$
\begin{array}{cc}
\varepsilon^{i}=\text { const., } & \kappa^{i}=0, \\
u^{i}=u^{i}(0)-\varepsilon^{i} x, & w^{i}=0, \\
\varphi^{i}=0, & x^{*}=x, \\
\Delta=0, & R_{X}^{i}=\mathcal{N}^{i}=\text { const. } \\
R_{Z}^{i}=\mathcal{Q}^{i}=0, & M_{Y}^{i}=\mathcal{M}^{i}=\text { const. } \\
p_{X}^{i}=p_{t}^{i}=0, & p_{Z}^{i}=p_{n}^{i}=0 .
\end{array}
$$

Finally, the linearized system of equilibrium Eqs. (2), (4)-(6), (8), (10), (14), and (16) when written at the primary configuration (18) of the composite column is easily derived in the following form: 


$$
\begin{gathered}
\delta \mathcal{F}_{1}=\delta u^{a \prime}-\delta \varepsilon^{a}=0, \\
\delta \mathcal{F}_{2}=\delta u^{b \prime}-\delta \varepsilon^{b}=0, \\
\delta \mathcal{F}_{3}=\delta w^{\prime}+(1+\varepsilon) \delta \varphi=0, \\
\delta \mathcal{F}_{4}=\delta \varphi^{\prime}-\delta \kappa=0, \\
\delta \mathcal{F}_{5}=\delta R_{X}^{a \prime}-\delta p_{t}=0, \\
\delta \mathcal{F}_{6}=\delta R_{X}^{b \prime}+\delta p_{t}=0, \\
\delta \mathcal{F}_{7}=\delta R_{Z}^{\prime}=0 \\
\delta \mathcal{F}_{8}=\delta M_{Y}^{\prime}+R_{X} \delta w^{\prime}-(1+\varepsilon) \delta R_{Z}-d \delta p_{t}=0, \\
\delta \mathcal{F}_{9}=\delta R_{X}^{a}-C_{11}^{a} \delta \varepsilon^{a}-C_{12}^{a} \delta \kappa=0, \\
\delta \mathcal{F}_{10}=\delta R_{X}^{b}-C_{11}^{b} \delta \varepsilon^{b}-C_{12}^{b} \delta \kappa=0, \\
\delta \mathcal{F}_{11}=\delta M_{Y}-C_{21}^{a} \delta \varepsilon^{a}-C_{21}^{b} \delta \varepsilon^{b}-\left(C_{22}^{a}+C_{22}^{b}\right) \delta \kappa=0, \\
\delta \mathcal{F}_{12}=\delta \Delta-\delta u^{a}+\delta u^{b}-d \delta \varphi=0, \\
\delta \mathcal{F}_{13}=\delta p_{t}-C_{33} \delta \Delta=0, \\
\delta \mathcal{F}_{14}=\delta x^{*}+\delta u^{a}-\delta x-\delta u^{b}-d \delta \varphi=0,
\end{gathered}
$$

where

$$
\begin{array}{ccc}
\delta w=\delta w^{a}=\delta w^{b}, & \delta \varphi=\delta \varphi^{a}=\delta \varphi^{b}, & \delta \kappa=\delta \kappa^{a}=\delta \kappa^{b}, \\
\delta R_{Z}=\delta R_{Z}^{a}+\delta R_{Z}^{b}, & \delta M_{Y}=\delta M_{Y}^{a}+\delta M_{Y}^{b}, & \delta p_{t}=\delta p_{t}^{a}=\delta p_{t}^{b},
\end{array}
$$

and

$$
\begin{gathered}
C_{11}^{i}=\frac{\partial \mathcal{N}_{C}^{i}}{\partial \varepsilon^{i}}=\int_{\mathcal{A}^{i}} \frac{\partial \sigma_{C}^{i}\left(\varepsilon^{i}, \kappa^{i}=0\right)}{\partial \varepsilon^{i}} \mathrm{~d} A^{i}=E_{t}^{i} A^{i}, \\
C_{12}^{i}=\frac{\partial \mathcal{N}_{C}^{i}}{\partial \kappa^{i}}=C_{21}^{i}=\frac{\partial \mathcal{M}_{C}^{i}}{\partial \varepsilon^{i}}=\int_{\mathcal{A}^{i}} z \frac{\partial \sigma_{C}^{i}\left(\varepsilon^{i}, \kappa^{i}=0\right)}{\partial \varepsilon^{i}} \mathrm{~d} A^{i}=E_{t}^{i} S^{i}, \\
C_{22}^{i}=\frac{\partial \mathcal{M}_{C}^{i}}{\partial \kappa^{i}}=\int_{\mathcal{A}^{i}} \frac{\partial \sigma_{C}^{i}\left(\varepsilon^{i}, \kappa^{i}=0\right)}{\partial \kappa^{i}} \mathrm{~d} A^{i}=E_{t}^{i} J^{i}, \\
C_{33}=\frac{\partial p_{t}}{\partial \Delta}=\frac{\partial \mathcal{H}}{\partial \Delta}=K,
\end{gathered}
$$


in which the quantities $C_{11}^{i}, C_{12}^{i}, C_{22}^{i}$, and $C_{33}$ are the components of the tangent constitutive matrix of the cross-sections of layer $a$ and $b$, and an interphase layer. In addition, $E_{t}^{i}$ denotes the uniaxial tangent modulus of the layer's material, $K$ is the initial contact stiffness, and $A^{i}, S^{i}$, and $J^{i}$ denote the cross-sectional area, static moment and moment of inertia of layers $a$ and $b$ with respect to the reference axis of the composite column, respectively.

Remark 1. It is, however, clear from the last equation of Eqs. (21) that $C_{33}$ is the initial stiffness of the interface force-slip law. Hence, the critical loads are dependent on the initial linear portion of the interface force-slip relation, but not on its general form.

Thus, Eqs. (19) constitute a linear system of 14 algebraic-differential equations of the first order with constant coefficients for 14 unknown functions of $x: \delta u^{i}, \delta w, \delta \varphi, \delta \varepsilon^{i}, \delta \kappa, \delta R_{X}^{i}, \delta R_{Z}, \delta M_{Y}, \delta p_{t}, \delta \Delta$, and $\delta x^{*}$ along with the corresponding natural and essential boundary conditions which may be written in the following general form, see e.g. [16, 19]:

$$
\begin{array}{ll}
s_{1}^{0} \delta R_{X}^{a}(0)+s_{2}^{0} \delta u^{a}(0)=0, & s_{1}^{L} \delta R_{X}^{a}(L)+s_{2}^{L} \delta u^{a}(L)=0, \\
s_{3}^{0} \delta R_{X}^{b}(0)+s_{4}^{0} \delta u^{b}(0)=0, & s_{3}^{L} \delta R_{X}^{b}(L)+s_{4}^{L} \delta u^{b}(L)=0, \\
s_{5}^{0} \delta R_{Z}(0)+s_{6}^{0} \delta w(0)=0, & s_{5}^{L} \delta R_{Z}(L)+s_{6}^{L} \delta w(L)=0, \\
s_{7}^{0} \delta M_{Y}(0)+s_{8}^{0} \delta \varphi(0)=0, & s_{7}^{L} \delta M_{Y}(L)+s_{8}^{L} \delta \varphi(L)=0,
\end{array}
$$

where $s_{i}^{0}, s_{i}^{L} \in\{0,1\}$ are parameters that determine different combinations of boundary conditions of the two-layer composite column. The superscript "0" and " $L$ " of $s$ identifies its value at $x=0$ and $x=L$, respectively.

Due to the fact that boundary conditions in the longitudinal and transverse direction are interrelated (see, [19]), the general solution of the system of linear 
algebraic-differential equations (19) is determined by nine integration constants $\mathcal{C}_{1}, \mathcal{C}_{2}, \mathcal{C}_{3}, \mathcal{C}_{4}, \mathcal{C}_{5}, \mathcal{C}_{6}, \mathcal{C}_{7}, \mathcal{C}_{8}$, and $\mathcal{C}_{9}$. These unknown integration constants are determined from the boundary conditions (22) and their combinations [19]. As a results, a system of nine homogeneous linear algebraic equations for nine unknown integration constants is obtained

$$
\mathbf{K}_{\mathrm{t}} \mathbf{c}=\mathbf{0},
$$

where $\mathbf{K}_{\mathbf{t}}$ and $\mathbf{c}$ denote a tangent matrix of the current equilibrium state on the fundamental path and a vector of unknown constants, respectively. For a non-trivial solution of (23), the determinant of the system matrix should vanish, see e.g. [25]

$$
\operatorname{det} \mathbf{K}_{\mathbf{t}}=0
$$

The condition (24) represents a linear eigenvalue problem and its solution, i.e. the lowest eigenvalue, corresponds to the smallest critical buckling load, $P_{c r}$, of the column. The condition (24) along with the boundary condition constitute a system of two non-linear algebraic equations

$$
\begin{gathered}
f_{1}\left(P_{\mathrm{cr}}, \varepsilon_{\mathrm{cr}}\right)=\operatorname{det} \mathbf{K}_{\mathbf{t}}=0, \\
f_{2}\left(P_{\mathrm{cr}}, \varepsilon_{\mathrm{cr}}\right)=\mathcal{N}^{a}+\mathcal{N}^{b}-P_{\mathrm{cr}}=0,
\end{gathered}
$$

for the two unknowns, i.e. the critical axial load, $P_{\mathrm{cr}}$, and the critical axial strain, $\varepsilon_{\mathrm{cr}}$. The system (25) is solved numerically using the Newton-Raphson iterative method.

\section{Comprehensive practical examples and discussion}

\subsection{Effect of non-linear interface compliance on critical buckling loads}

The analytical results, for critical buckling loads of geometrically perfect twolayer composite columns with interlayer slip, obtained herein with exact linear eigenvalue problem will be employed to analyze the non-linear interface 
compliance on critical buckling loads. With the intention of analyzing the above-mentioned effect, an inelastic two-layer composite column is employed. Geometrical and mechanical properties of the two-layer composite column are presented in Fig. 3.

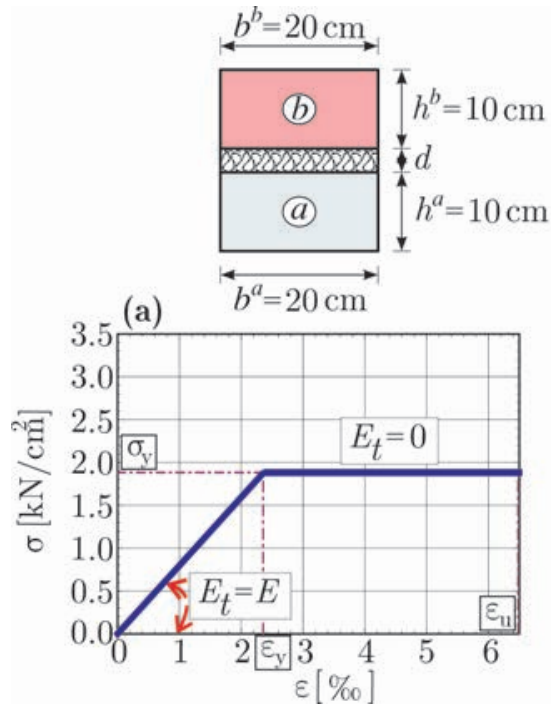

(a) Timber

(b) Timber

$\frac{L}{h^{a}+h^{b}}=25$

$E^{a}=800 \mathrm{kN} / \mathrm{cm}^{2}$

$E^{b}=800 \mathrm{kN} / \mathrm{cm}^{2}$

$\varepsilon_{\mathrm{y}}=-\frac{200}{85} \% \quad \varepsilon_{\mathrm{u}}=-\frac{65}{10} \%$

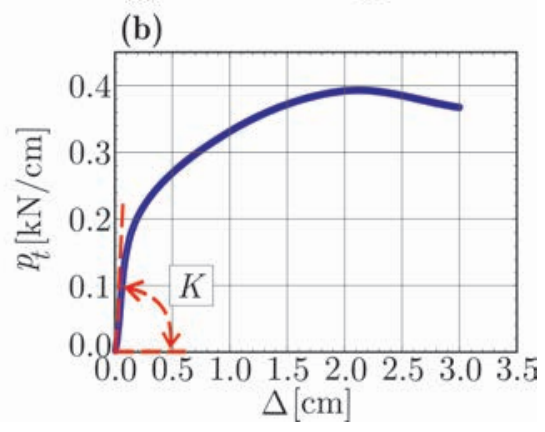

Figure 3. Geometrical and mechanical properties of the two-layer composite column;

(a) idealized bi-linear stress-strain constitutive law of timber [28]; (b) non-linear constitutive law of the contact [14].

The critical buckling loads of the two-layer composite column with different types of end conditions have been evaluated. Four sets of boundary conditions have been considered of practical importance for columns with nonmovable supports: clamped-free column (C-F), clamped-clamped column (C-C), clamped-pinned column (C-P) and pinned-pinned column (P-P). In accordance to the boundary conditions (22) the classical boundary conditions of two-layer Euler columns and the corresponding non-zero values of parameters $s_{i}^{0}, s_{i}^{L}$, and effective length coefficient, $\beta_{\mathrm{E}}$, are summarized in Table 1.

The critical buckling loads have been calculated for different types of boundary conditions, different values of an interlayer stiffness, $K$, and various thicknesses 
Table 1

Classical two-layer column boundary conditions and effective length coefficients $\beta_{E}$ of solid Euler columns.

\begin{tabular}{|c||c|c|c|c|}
\hline \hline Classical cases & C-F & C-C & C-P & P-P \\
\hline \hline & $s_{2}^{0}=s_{4}^{0}=1$ & $s_{2}^{0}=s_{4}^{0}=1$ & $s_{2}^{0}=s_{4}^{0}=1$ & $s_{2}^{0}=s_{4}^{0}=1$ \\
Non-zero values & $s_{6}^{0}=s_{8}^{0}=1$ & $s_{6}^{0}=s_{8}^{0}=1$ & $s_{6}^{0}=s_{8}^{0}=1$ & $s_{6}^{0}=s_{7}^{0}=1$ \\
$s_{i}^{0}$ and $s_{i}^{L}$ & $s_{1}^{L}=s_{3}^{L}=1$ & $s_{1}^{L}=s_{3}^{L}=1$ & $s_{1}^{L}=s_{3}^{L}=1$ & $s_{1}^{L}=s_{3}^{L}=1$ \\
& $s_{5}^{L}=s_{7}^{L}=1$ & $s_{6}^{L}=s_{8}^{L}=1$ & $s_{6}^{L}=s_{7}^{L}=1$ & $s_{6}^{L}=s_{7}^{L}=1$ \\
\hline \multirow{2}{*}{$\begin{array}{c}\text { Effective length } \\
\text { coefficients }\end{array}$} & $\beta_{\mathrm{E}}=2$ & $\beta_{\mathrm{E}}=0.5$ & $\beta_{\mathrm{E}}=0.699$ & $\beta_{\mathrm{E}}=1$ \\
\hline \hline
\end{tabular}

$\mathrm{C}=$ clamped (fixed); $\mathrm{F}=$ free; $\mathrm{P}=$ pinned

of an interface layer, $d$. The results are shown graphically in Figs. 4-6.

In Fig. 4, a critical buckling load of C-F two-layer composite column, $P_{\mathrm{cr}}$, normalized with respect to the critical buckling force of the corresponding solid Euler column, $P_{\mathrm{E}}$, is shown for various inter-layer slip moduli, $K$ and, different thicknesses of an interfacial layer, $d$. Namely, $\bar{P}_{\mathrm{cr}}=\frac{P_{\mathrm{cr}}(d \neq 0)}{P_{\mathrm{E}}(d \neq 0)}$.

It is clear from Fig. 4 that by increasing the thickness of the interfacial layer, $d$, the normalized critical buckling load $\bar{P}_{\text {cr }}$ decreases. On the other hand, the normalized critical buckling load $\bar{P}_{\text {cr }}$ increases with increase of inter-layer slip modulus, $K$. For instance, in the limiting case when there is an absolutely stiff connection $(\delta \Delta=0 ; K \rightarrow \infty), \bar{P}_{\mathrm{cr}}[d=0 \mathrm{~cm}]=1.0003 ; \bar{P}_{\mathrm{cr}}[d=1 \mathrm{~cm}]=0.8642$; $\bar{P}_{\text {cr }}[d=2 \mathrm{~cm}]=0.7521 ; \bar{P}_{\text {cr }}[d=3 \mathrm{~cm}]=0.6592 ;$ or there exists no connection between the layers $\left(\delta \Delta=\delta \Delta_{\max } \neq 0 ; K \rightarrow 0\right), \bar{P}_{\mathrm{cr}}[d=0 \mathrm{~cm}]=0.2503$; $\bar{P}_{\mathrm{cr}}[d=1 \mathrm{~cm}]=0.2160 ; \bar{P}_{\mathrm{cr}}[d=2 \mathrm{~cm}]=0.1880 ; \bar{P}_{\mathrm{cr}}[d=3 \mathrm{~cm}]=0.1648$. Evidently, by increasing the inter-layer slip modulus, $K$, the exact buckling load 


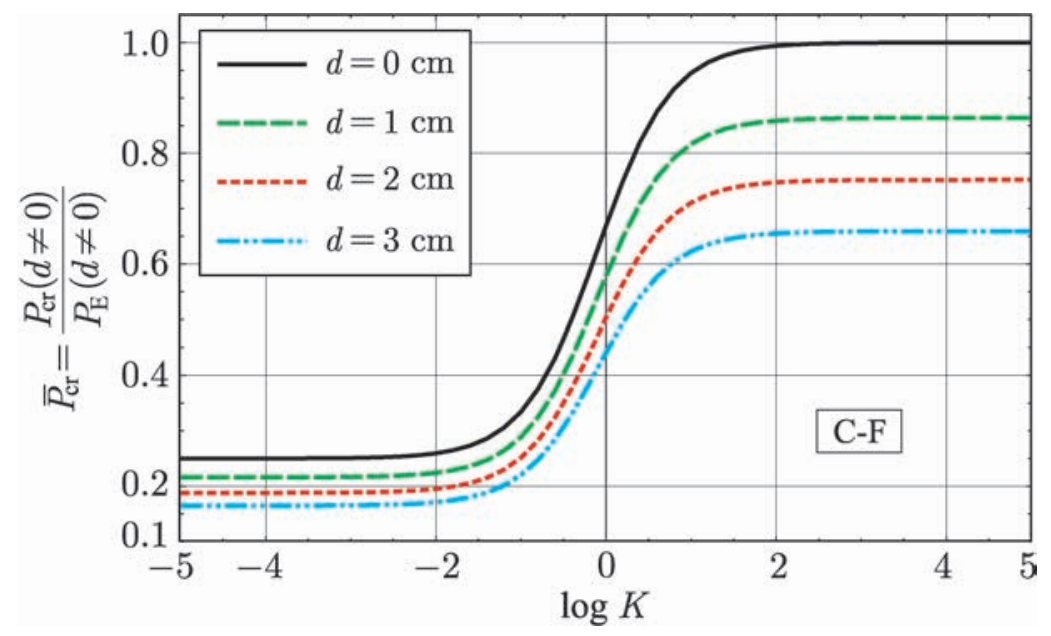

Figure 4. Normalized critical buckling load, $\bar{P}_{\mathrm{cr}}$, of geometrically perfect C-F two-layer composite column for different thicknesses of interfacial layer, $d$ and, different $K \mathrm{~s}$.

of geometrically perfect two-layer composite column, $P_{\mathrm{cr}}$, converges perfectly to the buckling load of the corresponding Euler solid column, $P_{\mathrm{E}}$, only for $d=0 \mathrm{~cm}$.

Furthermore, in Fig. 5, the same plot for C-P two-layer composite column is shown. Note that for the limiting case, the very resembling results to the results for C-F two-layer composite column have been obtained.

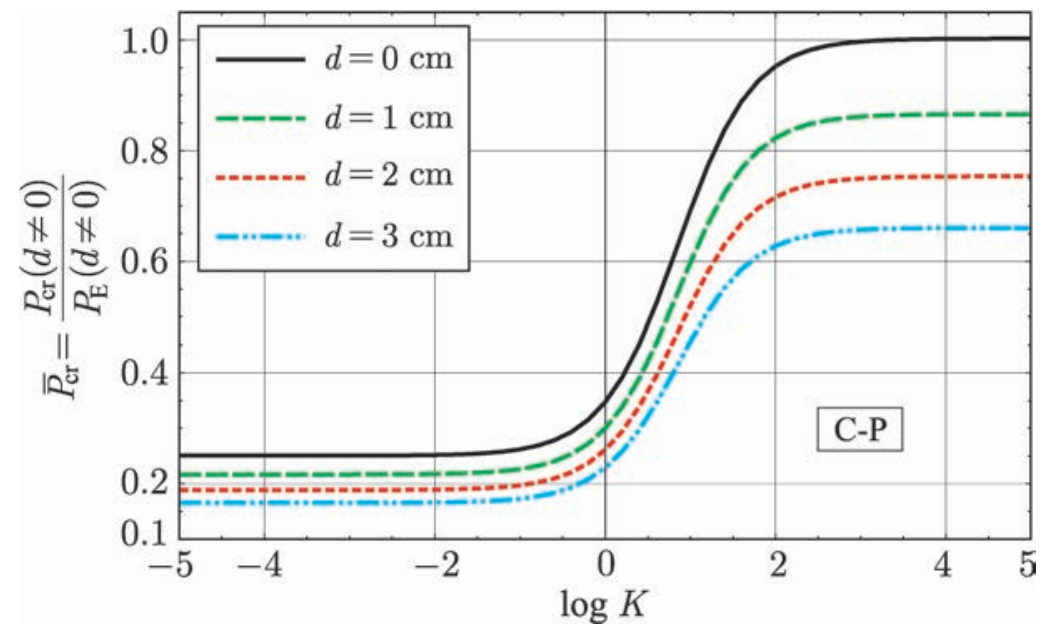

Figure 5. Normalized critical buckling load, $\bar{P}_{\text {cr }}$, of geometrically perfect C-P two-layer composite column for different thicknesses of interfacial layer, $d$ and, different $K \mathrm{~s}$. 
In addition, from Figs. 4 and 5 , it is clear that the distribution of $\bar{P}_{\mathrm{cr}}$ versus interlayer modulus $K$ is different for different values of $d$ and various types of boundary conditions. Thus, for $K=1 \mathrm{kN} / \mathrm{cm}^{2}(\log K=0), \bar{P}_{\mathrm{cr}}[d=0 \mathrm{~cm}]=$ $0.6692 ; \bar{P}_{\mathrm{cr}}[d=1 \mathrm{~cm}]=0.5782 ; \bar{P}_{\mathrm{cr}}[d=2 \mathrm{~cm}]=0.5032 ; \bar{P}_{\mathrm{cr}}[d=3 \mathrm{~cm}]=$ 0.4410 in case of C-F column, while, on the other hand, in case of C-P column $\bar{P}_{\mathrm{cr}}[d=0 \mathrm{~cm}]=0.3477 ; \bar{P}_{\mathrm{cr}}[d=1 \mathrm{~cm}]=0.3003 ; \bar{P}_{\mathrm{cr}}[d=2 \mathrm{~cm}]=0.2614 ;$ $\bar{P}_{\mathrm{cr}}[d=3 \mathrm{~cm}]=0.2291$. Since, the distributions of $\bar{P}_{\mathrm{cr}}$ for $\mathrm{C}-\mathrm{C}$ composite column are very similar to distributions in case of C-F and C-P composite column, the results are not described in this paper.

When treating various structural stability problems it is often useful to express the buckling load, $P_{\mathrm{cr}}$, in the form of the Euler formula with a suitable modification of the column length. Thus, the critical load of a layered geometrically perfect composite column with an interlayer slip may be expressed in terms of the classical Euler formula for a solid column as

$$
P_{\mathrm{cr}}=\frac{\pi^{2}(E J)_{s}}{\left(\beta_{\mathrm{cr}} L\right)^{2}}
$$

in which $(E J)_{s}$ is the flexural rigidity of the corresponding solid column and $\beta_{\text {cr }}$ denotes the critical effective length parameter of the geometrically perfect two-layer composite column which depends entirely on the particular buckling mode, inter-layer contact stiffness, $K$, and layer's thickness, $d$ and, should not be confused with the effective length coefficient, $\beta_{\mathrm{E}}$, that gives the distance between the points of inflection in a solid column. The effective length coefficient, $\beta_{\mathrm{cr}}$, is obtained by a comparison of the critical force, $P_{\mathrm{cr}}$, calculated with the presented exact model and the Euler critical force, $P_{\mathrm{E}}$, for a corresponding 
solid column

$$
\beta_{\mathrm{cr}}=\sqrt{\frac{P_{\mathrm{E}}}{P_{\mathrm{cr}}}} \beta_{\mathrm{E}} .
$$

The critical effective length coefficient, $\beta_{\mathrm{cr}}$, against the layer's thickness, $d$, is shown in Figs. 6 and 7 for different $K$ s and two kinds of end conditions, such as C-F and C-P two-layer composite columns. From Fig. 6 it can be seen

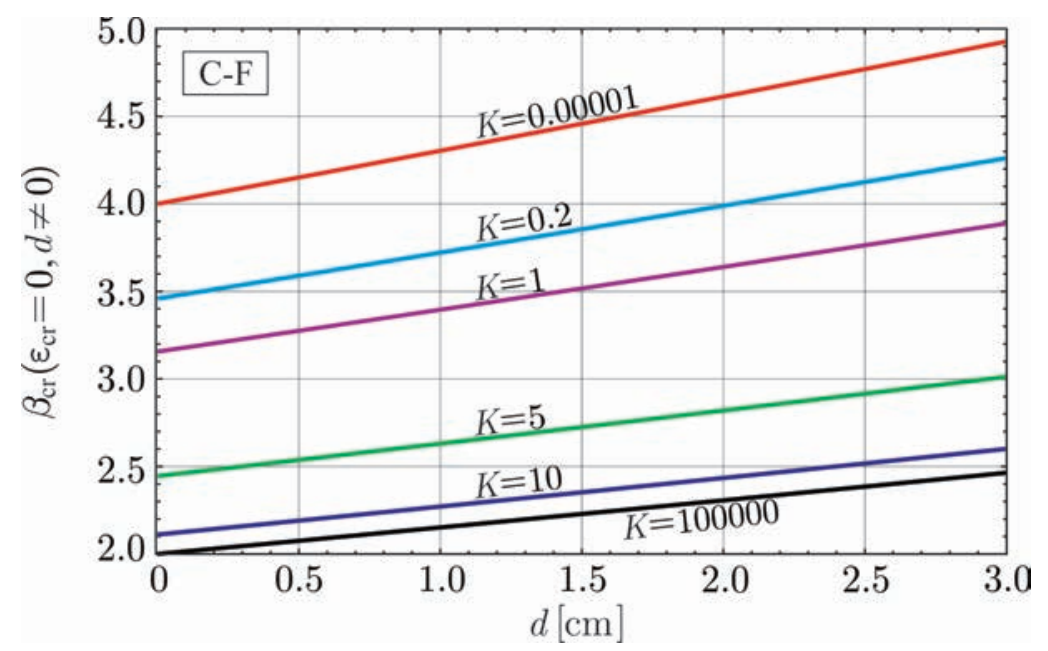

Figure 6. Illustrations of critical effective length parameter, $\beta_{\mathrm{cr}}$, in case of C-F composite column for different $K$ s and $d$.

that $\beta_{\text {cr }}$ is higher for lower values of $K$ and higher values of $d$. The effect of $d$ on the $\beta_{\text {cr }}$ becomes less pronounced for higher values of $K$. For example, when $K=0.2 \mathrm{kN} / \mathrm{cm}^{2}, \beta_{\mathrm{cr}}[d=0 \mathrm{~cm}]=3.1558 ; \beta_{\mathrm{cr}}[d=1 \mathrm{~cm}]=3.3953$; $\beta_{\mathrm{cr}}[d=2 \mathrm{~cm}]=3.6395 ; \beta_{\mathrm{cr}}[d=3 \mathrm{~cm}]=3.8876$, while, for $K=10 \mathrm{kN} / \mathrm{cm}^{2}$, $\beta_{\mathrm{cr}}[d=0 \mathrm{~cm}]=2.0569 ; \beta_{\mathrm{cr}}[d=1 \mathrm{~cm}]=2.2130 ; \beta_{\mathrm{cr}}[d=2 \mathrm{~cm}]=2.3722 ;$ and $\beta_{\mathrm{cr}}[d=3 \mathrm{~cm}]=2.5339$. Furthermore, if there exist no interface layer between the layers, e.i. $d=0$, the $\beta_{\text {cr }}$ is in case of a fully flexible connection $\left(K=10^{-5} \mathrm{kN} / \mathrm{cm}^{2}\right)$ two times higher than in the absolute stiff connection case. On the other hand, in case of a rigid connection $\left(K=10^{5} \mathrm{kN} / \mathrm{cm}^{2}\right), \beta_{c r}$ almost equals $\beta_{\mathrm{E}}$. In this case, the critical load of two-layer composite column is identical with the critical load of the corresponding solid column. 
Fig. 7 gives the $\beta_{\text {cr }}$ versus $d$ response for different values of $K$ for C-P boundary conditions of the two-layer composite column. Likewise as in the C-F boundary conditions case, the effect of $d$ on the $\beta_{\text {cr }}$ in this case becomes less pronounced for higher values of $K$. For example, when $K=0.2 \mathrm{kN} / \mathrm{cm}^{2}, \beta_{\mathrm{cr}}[d=0 \mathrm{~cm}]=$ $1.3397 ; \beta_{\mathrm{cr}}[d=1 \mathrm{~cm}]=1.4413 ; \beta_{\mathrm{cr}}[d=2 \mathrm{~cm}]=1.5450 ; \beta_{\mathrm{cr}}[d=3 \mathrm{~cm}]=1.6503$, while, for $K=10 \mathrm{kN} / \mathrm{cm}^{2}, \beta_{\text {cr }}[d=0 \mathrm{~cm}]=0.8405 ; \beta_{\text {cr }}[d=1 \mathrm{~cm}]=0.9043$; $\beta_{\mathrm{cr}}[d=2 \mathrm{~cm}]=0.9693 ;$ and $\beta_{\mathrm{cr}}[d=3 \mathrm{~cm}]=1.0354$.

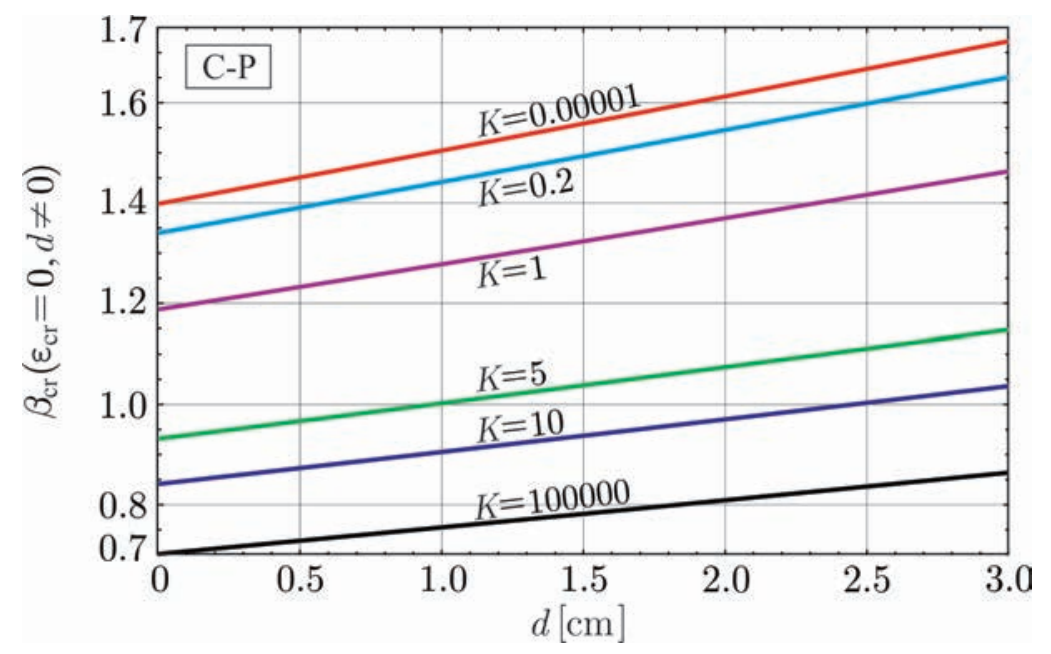

Figure 7. Illustrations of critical effective length parameter, $\beta_{\mathrm{cr}}$, in case of C-P composite column for different $K \mathrm{~s}$ and $d$.

\subsection{Effect of material inelasticity on critical buckling loads}

In order to simulate and investigate the inelastic buckling behavior of a P-P two-layer composite column, the critical buckling loads have been calculated for geometric and material data given in Fig. 8. These loads are further normalized by the ultimate axial load-carrying capacity, $P_{\mathrm{ult}}$, and plotted as a function of column slenderness, $\lambda$, in Fig. 9. Here the column slenderness, $\lambda$, is defined as

$$
\lambda=\frac{\beta_{E} L \sqrt{A^{a}+A^{b}}}{\sqrt{I^{a}+I^{b}}} .
$$


Variation in the column slenderness was achieved by considering a range of column lengths.

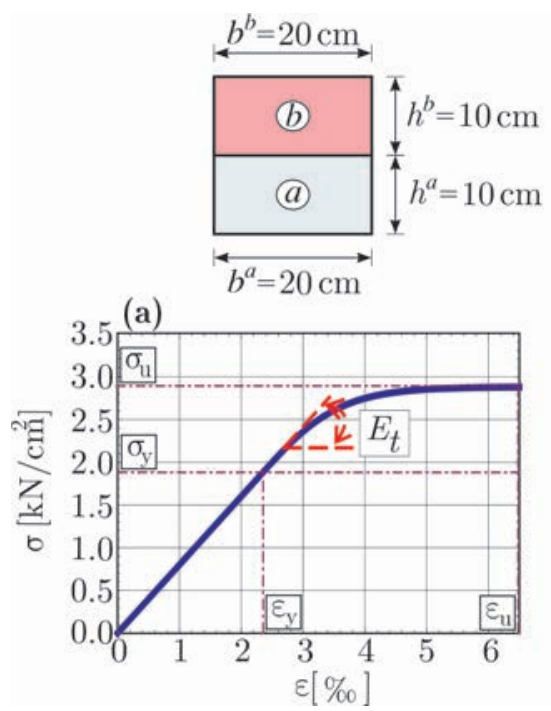

(a) Timber

(b) Timber

$\frac{L}{h^{a}+h^{b}}=25$

$E^{a}=800 \mathrm{kN} / \mathrm{cm}^{2}$

$E^{b}=800 \mathrm{kN} / \mathrm{cm}^{2}$

$\varepsilon_{y}=-\frac{200}{85} \% \quad \varepsilon_{\mathrm{u}}=-\frac{65}{10} \%$

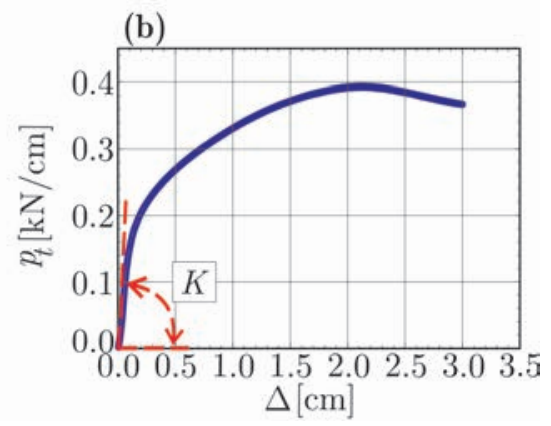

Figure 8. Geometrical and mechanical properties of the composite column; $(a)$ inelastic stress-strain constitutive law of timber [28]; $(b)$ non-linear constitutive law of the contact [14].

Note that the difference between the elastic and inelastic buckling loads decreases significantly as the column becomes more slender. Correspondingly, at the specified column slenderness ratio $\lambda_{\mathrm{tr}}^{2} \approx 38$, there is a smooth transition between the elastic and inelastic buckling. For $\lambda>\lambda_{\mathrm{tr}}^{2}$, the elastic buckling occurs and the column is treated as slender.

On the other hand, the transition between the crushing of the material and inelastic buckling occurs at $\lambda_{\text {tr }}^{1} \approx 3$. For $\lambda<\lambda_{\text {tr }}^{2}$, only the crushing of the material occurs and the column is called short or stocky. Otherwise, for $\lambda_{\mathrm{tr}}^{1}<$ $\lambda<\lambda_{\text {tr }}^{2}$, the column is called intermediate. For example, in this intermediate inelastic region, the normalized inelastic buckling load, i.e. $P_{\mathrm{cr}}^{*}=\frac{P_{\mathrm{cr}}(d=0)}{P_{\mathrm{ult}}(d=0)}$, reads as $P_{\mathrm{cr}}^{*}[\lambda=12]=0.9720 ; P_{\mathrm{cr}}^{*}[\lambda=19]=0.9274 ; P_{\mathrm{cr}}^{*}[\lambda=26]=0.8601 ;$ and $P_{\mathrm{cr}}^{*}[\lambda=33]=0.7635$. Besides, it is seen from Fig. 9 that the prevalent 


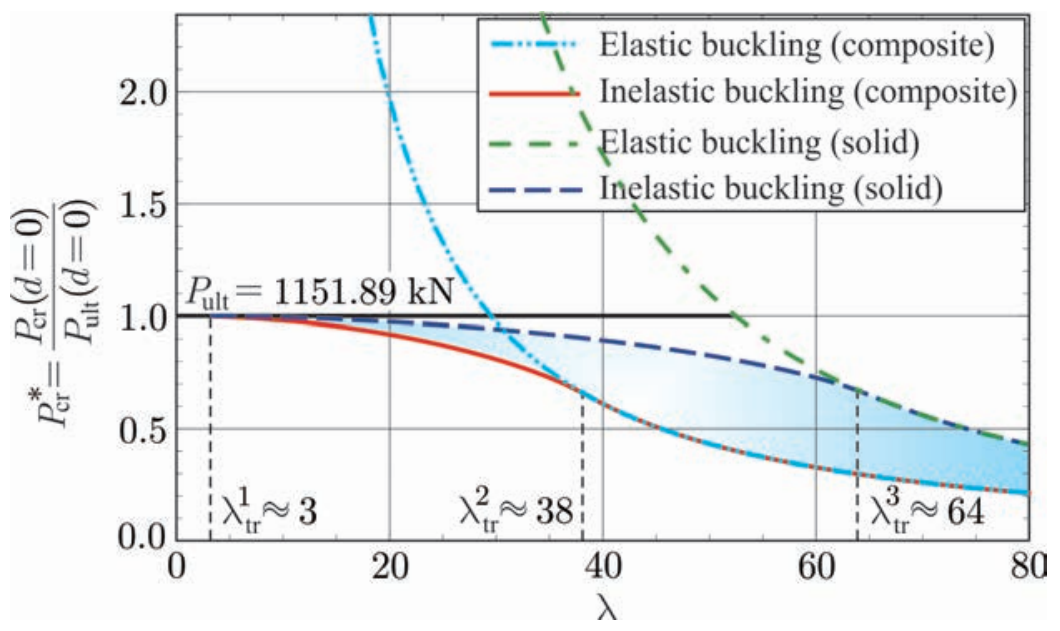

Figure 9. Non-dimensional column elastic and inelastic buckling curves for solid and two-layer composite columns.

failure mode of the two-layer column is buckling and not the crushing of the material. It is also seen that the effects of material inelasticity decreases with increasing the column slenderness and becomes much less pronounced for slender columns, as shown in Fig. 9.

In addition, the normalized critical buckling loads of the corresponding solid column are calculated and shown in Fig. 9. It is seen that the interlayer stiffness, $K$, has a significant influence on critical buckling loads and the type of buckling. For example, when $\lambda=50$, the normalized inelastic buckling load of the composite column, $P_{\mathrm{cr}}^{*}\left[K=1 \mathrm{kN} / \mathrm{cm}^{2}\right]$, is 0.4320 , while, the equivalent normalized buckling load of a solid column is $P_{\mathrm{cr}}^{*}\left[K=\infty \mathrm{kN} / \mathrm{cm}^{2}\right]=0.8267$. It is also apparent from Fig. 9 that in this particular case, the influence of interlayer stiffness, $K$, on inelastic buckling loads, is the highest for column slenderness ratios approximately between 40 and 70. As was already noted, the interlayer stiffness, $K$, has a significant effect on the transition between elastic and inelastic buckling. Thus, for a solid column, the transition column slenderness, $\lambda_{\mathrm{tr}}^{3}$, is always higher than for a composite column. In this particular case, $\lambda_{\mathrm{tr}}^{3} \approx 64$, which is almost twice as much as in case of the composite 
column where $\lambda_{\text {tr }}^{2} \approx 38$.

\section{Conclusions}

A mathematical model for the slip-buckling problem has been proposed and its exact solution has been found for the analysis of materially inelastic geometrically perfect two-layer composite columns with non-linear compliance between the layers. The mathematical model has been applied to evaluate exact critical buckling forces. For this purpose, a parametric study has been performed by which the influence of various material and geometric parameters on buckling forces have been investigated. The theoretical derivation, the numerical computations and the analysis of the subsequent results have led to the following conclusions:

(1) It has been mathematically exactly derived that the critical buckling loads are influenced by the initial stiffness only, and hence on linear portion of the interface force-slip relation, but not on its general form.

(2) The results have confirmed that the reduced stiffness between the layers can promote buckling which can lead to a drastic reduction of the bifurcation loads.

(3) The influence of interlayer thickness on the exact critical buckling forces is boundary conditions dependent.

(4) The material inelasticity can have an inportant influence on critical buckling loads and thus especially in case of short columns should not be neglected.

(5) The interlayer stiffness has a significant effect on the transition between the elastic and inelastic buckling. The transition slenderness of a solid 
column is always higher than for a composite column.

(6) The timber composite columns fail predominantly by buckling and not by crushing of the material.

\section{Acknowledgement}

The authors acknowledge the financial support from the state budget by the Slovenian Research Agency (project No. Z2-2031).

\section{References}

[1] Dall'Asta, A., Zona, A., 2004. Comparison and validation of displacement and mixed elements for the non-linear analysis of continuous composite beams. Computers and Structures 82(23-26) 2117-2130.

[2] Battini, J.M., Nguyen, Q.H., Hjiaj, M., 2009. Non-linear finite element analysis of composite beams with interlayer slips. Computers and Structures 87 904-912.

[3] Čas, B., Saje, M., Planinc, I., 2007. Buckling of layered wood columns. Advances in Engineering Software 38 586-597.

[4] Silva, A.R., Sousa, J.B.M., 2009. A family of interface elements for the analysis of composite beams with interlayer slip. Finite Elements in Analysis and Design 45(5) 305-314.

[5] Heuer, R., 2004. Equivalence of the analyses of sandwich beams with or without interlayer slip. Mechanics of Advanced Materials and Structures $11425-432$.

[6] Kroflič, A., Planinc, I., Saje, M., Turk, G., Čas, B., 2010. Non-linear analysis of two-layer timber beams considering interlayer slip and uplift. Engineering Structures, 32(6), 1617-1630.

[7] Faella, C., Martinelli, E., Nigro, E., 2009. Steel-concrete composite beams 
in partial interaction: Closed-form "exact" expression of the stiffness matrix and the vector of equivalent nodal forces. Engineering Structures, 32(9) $2744-2754$.

[8] Challamel, N., 2009. On lateral-torsional vibrations of elastic composite beams with interlayer slip. Journal of Sound and Vibration 325(4-5) 10121022.

[9] Ranzi, G., 2008. Locking problems in the partial interaction analysis of multi-layered composite beams. Engineering Structures 30(10) 2900-2911.

[10] Ranzi, G., Dall'Asta, A., Ragini, L., Zona, A., 2010. A geometric nonlinear model for composite beams with partial interaction. Engineering Structures 32(5) 1384-1396.

[11] Schnabl, S., Planinc, I., Saje, M., Čas, B. and Turk, G., 2006. An analytical model of layered continuous beams with partial interaction. Structural Engineering and Mechanics 22 (3) 263-278.

[12] Schnabl, S., Saje, M., Turk, G. and Planinc, I., 2007. Locking-free twolayer Timoshenko beam element with interlayer slip. Finite Elements in Analysis and Design 43 705-714.

[13] Schnabl, S., Saje, M., Turk, G. and Planinc, I., 2007. Analytical solution of two-layer beam taking into account interlayer slip and shear deformation. Journal of Structural Engineering ASCE 133(6) 886-894.

[14] Planinc, I., Schnabl, S., Saje, M., Lopatič, J. and Čas, B., 2008. Numerical and experimental analysis of timber composite beams with interlayer slip. Engineering Structures 30(11) 2959-2969.

[15] Girhammar, U.A., Pan, D.H., 2007. Exact static analysis of partially composite beams and beam-columns. International Journal of Mechanical Sciences 49(2) 239-255.

[16] Kryžanowski, A., Schnabl, S., Turk, G., Planinc, I., 2009. Exact slip- 
buckling analysis of two-layer composite columns. International Journal of Solids and Structures 46 2929-2938.

[17] Rassam, H.Y, Goodman, J.R., 1970. Buckling behaviour of layered wood columns. Wood Science 2(4) 238-246.

[18] Xu, R., Wu, Y., 2007. Static, dynamic, and buckling analysis of partial interaction composite members using Timoshenko's beam theory. International Journal of Mechanical Sciences 49(10) 1139-1155.

[19] Schnabl, S., Planinc, I., 2010. The influence of boundary conditions and axial deformability on buckling behavior of two-layer composite columns with interlayer slip. Engineering Structures 32(10) 3103-3111.

[20] Schnabl, S., Planinc, I., 2011. The effect of transverse shear deformation on the buckling of two-layer composite columns with interlayer slip. International Journal of Non-Linear Mechanics 46(3) 543-553.

[21] Foraboschi, P., 2009. Analytical solution of two-layer beam taking into account nonlinear interlayer slip. Journal of Engineering Mechanics 135(10) $1129-1146$.

[22] Keller, H.B., 1970. Nonlinear bifurcation. Journal of Differential Equations 7 417-434.

[23] Flajs, R., Saje, M., Zakrajšek, E., 2003. On the existence and uniqueness of the generalized solution of the Reissner's elastica. Mathematics and Mechanics of Solids 8 3-19.

[24] Hartmann, F., 1985. The Mathematical Foundation of Structural Mechanics. Springer-Verlag Berlin and Heidelberg GmbH \& Co.K.

[25] Planinc, I., Saje, M., 1999. A quadratically convergent algorithm for the computation of stability points: The application of the determinant of the tangent stiffness matrix. Computer Methods in Applied Mechanics and Engineering 169 89-105. 
[26] Reissner, E., 1972. On one-dimensional finite-strain beam theory: The plane problem. Journal of Applied Mechanics and Physics (ZAMP) 23 $795-804$.

[27] Vratanar, B., Saje, M., 1999. A consistent equilibrium in a cross-section of an elastic-plastic beam. International Journal of Solids and Structures $36311-337$.

[28] R. Pischl, Holzbau mit kritischen betractungen und neuen vorschlägen zur bemessung nach theorie 1. und 2. ordnung, Holzbau: Technische Universität Graz, Fakultät für Bauingenierwesen, 1980. 